\title{
Online monitoring of voltage stability margin using PMU measurements
}

\author{
Pankaj Sahu, M. K. Verma \\ Department of Electrical Engineering, Indian Institute of Technology (BHU), India
}

\begin{abstract}
Article Info
Article history:

Received Apr 15, 2019

Revised Oct 11, 2019

Accepted Oct 20, 2019

\section{Keywords:}

Critical bus

Online monitoring

Phasor measurements units

Voltage stability

Wide area monitoring system

ABSTRACT

With the growing smart grid concept it becomes important to monitor health of the power system at regular intervals for its secure and reliable operation. Phasor Measurement Units (PMUs) may play a vital role in this regard. This paper presents voltage stability monitoring in real time framework using synchrophasor measurements obtained by PMUs. Proposed approach estimates real power loading margin as well as reactive power loading margin of most critical bus using PMU data. As system operating conditions keep on changing, loading margin as well as critical bus information is updated at regular intervals using fresh PMU measurements. Simulations have been carried out using Power System Analysis Toolbox (PSAT) software. Accuracy of proposed Wide Area Monitoring System (WAMS) based estimation of voltage stability margin has been tested by comparing results with loading margin obtained by continuation power flow method (an offline approach for accurate estimation of voltage stability margin) under same set of operating conditions. Case studies performed on IEEE 14-bus system, New England 39-bus system and a practical 246-bus Indian power system validate effectiveness of proposed approach of online monitoring of loading margin.
\end{abstract}

Copyright $(2020$ Institute of Advanced Engineering and Science. All rights reserved.

Corresponding Author:

Pankaj Sahu,

Department of Electrical Engineering,

Indian Institute of Technology (BHU),

Varanasi 221005, India.

Email: psahu.rs.eee13@itbhu.ac.in

\section{INTRODUCTION}

Voltage stability has been considered as an important threat against secure operation of power system [1]. Several incidences of voltage instability initiated grid failures have been observed in different parts of the world [2]. Various approaches for offline estimation of voltage stability have been well documented [3]. Offline assessment of voltage stability is quite useful in advance planning of preventive and corrective measures against instability. However, secure operation of a system in real time framework requires its online monitoring against instability. A forecasting-aided state estimation has been proposed for online monitoring of voltage stability [4]. Online assessment of voltage stability margin based on available reactive power reserve has been suggested [5]. Yiwei Qiu et. al. proposed parametric polynomial approximation of static voltage stability region boundaries based on Galerkin method and, suggested real time determination of left and right eigen vectors associated with zero eigen value at the estimated saddlenode-bifurcation space for online monitoring and control of voltage stability [6].

With advancement in wide-area monitoring system (WAMS) technology, online monitoring of voltage stability through time stamped measurements by Phasor Measurement Units (PMUs) seems possible. In comparison to Supervisory Control and Data Acquisition (SCADA) System the utilization of PMUs shows improved decision making and operation [7, 8]. Many researchers proposed online monitoring of voltage stability margin by obtaining Thevenin's equivalence of network across a critical load bus based on real time 
measurements by PMUs. Online estimation of voltage stability margin through matching of critical load impedance with PMU measurements based Thevenin's impedance of the rest of the network has been proposed [9-12]. Representation of whole network connected across a critical load bus may be suitable for voltage stability monitoring of radial networks. However, interconnected power system may have a critical area comprising of a set of critical load buses prone to voltage collapse. Thevenin's equivalent of critical load area based on PMU measurements at its surrounding buses has been proposed [13, 14]. All the buses in the critical load area have been merged to replace these by a fictitious load bus. A critical load area is fed by multiple tie-lines, in general. Some of these may have over flows that may lead to voltage instability in the area. Replacing all the buses in the area with a single equivalent bus merges all the tie-lines too into a fictitious equivalent tie-line. Therefore, tie-lines of original network having overflows and hence being responsible for instability cannot be detected. In order to address this issue, online monitoring of voltage stability margin of a load area based on tie-line flows has been proposed [15]. Tie-line flows have been obtained through phasor measurements performed by PMUs placed at boundary buses of the critical load area. However, critical load areas are dependent upon operating conditions and topology of the network. Change of network topology due to occurrence of contingencies may lead to emergence of new critical load areas where PMUs are not placed.

Distributed linear algorithm has been proposed for online computation of voltage stability proximity indices (VCPI) based on local phasor measurements performed at all the load buses [16]. PMU measurements based online monitoring of critical buses using Q-V (reactive power-voltage magnitude) and P- $\Theta$ (real power-voltage angle) sensitivities has been proposed [17]. However, assumption of Q- $\Theta$ and $\mathrm{P}-\mathrm{V}$ decoupling are not valid near nose point. A normalized P-index has been proposed for online monitoring of voltage stability using phasor measurements [18]. However, P-index has been developed assuming constant power factor under increased demand which is not valid for real time systems.

Online monitoring of voltage stability based on Thevenin's equivalent of the network [9-15], as well as sensitivity based real time estimation of voltage stability margin [16-18] may fail to produce satisfactory results in case of large disturbances due to highly non-linear behavior of power systems. An Artificial Neural Network (ANN) based monitoring of voltage stability based on phasor measurements has been proposed [19]. Proper training of ANN is still a challenge. Enhanced-Online-Random-Forest (EORF) model has been proposed based on voltage phasor measurements for online monitoring of voltage stability [20]. EORF model updates voltage stability information under change in operating conditions/network topology using fresh PMU measurements at important load buses. EORF model may lead sometimes to erroneous estimation of voltage stability margin due to non-consideration of voltage phasor information of remaining buses. A general method to adjust loads at the receiving end has been applied to determine the proximity to voltage collapse [21]. Here, it is concluded that the intermediate load adjustment improves the accuracy of the indices. In this the PMUs are not placed optimally in the system. In most of the research the statistical information obtained from PMUs has not actionably used to improve the voltage stability. In [22], the new method has been suggested that gauges and improves the voltage stability of a system using statistical data obtained from PMUs.

In this paper, real time determination of nose curve of all the load buses based on three successive PMU measurements and pseudo-measurements is performed. Minimum out of maximum loadability of all the load buses has been considered as the loading margin of the system. Voltage stability information is updated with new PMU measurements obtained. Thus, proposed approach is capable of monitoring voltage stability of real time systems as change in system operating conditions and network topology is considered by updated PMU measurements performed at regular intervals. PMUs have been optimally placed in the system based on result of binary integer linear programming ensuring full network observability even in case of loss of few PMUs under contingencies [23]. Paper is structured as follows: Section II presents methodology proposed for online monitoring of voltage stability margin using PMU measurements based quadratic fitting of nose curves. Section III validates effectiveness of proposed approach based on case studies performed on standard IEEE 14-bus system, New England 39-bus system and a practical 246-bus Indian system representing power network of nine states and union territories of India. Section IV concludes summarizing important contributions of the proposed work.

\section{METHODOLOGY}

Proposed approach of online monitoring of voltage stability margin using phasor measurements is presented: Real power demand $\left(P_{D_{i}}\right)$ versus voltage magnitude $V_{i}$ curve $(P-V$ curve) of bus- $i$ shown in Figure 1 may be approximately obtained by solution of quadratic equation, 


$$
P_{D_{i}}=a_{1 i} V_{i}^{2}+a_{2 i} V_{i}+a_{3 i}
$$

where, $a_{1 i}, a_{2 i}$ and $a_{3 i}$ are constants

$$
\begin{aligned}
& \text { Differentiating } P_{D_{i}} \text { with respect to } V_{i}, \\
& \frac{d P_{D_{i}}}{d V_{i}}=2 a_{1 i} V_{i}+a_{2 i}
\end{aligned}
$$

At nose point of $P$-V curve, $\frac{d P_{D_{i}}}{d V_{i}}=0$. Therefore, from (2),

$$
V_{i}^{n p}=-\frac{a_{2 i}}{2 a_{1 i}}
$$

where, $V_{i}^{n p}=$ voltage magnitude of bus $-i$ at the nose point of $P-V$ curve shown in Figure1. From (1) and (3),

$$
P_{D_{i}}^{n}=-\frac{a_{2 i}^{2}}{4 a_{1 i}}+a_{3 i}
$$

where, $P_{D_{i}}^{n}=$ Real power demand of bus $-i$ at the nose point of $P-V$ curve shown in Figure 1 .

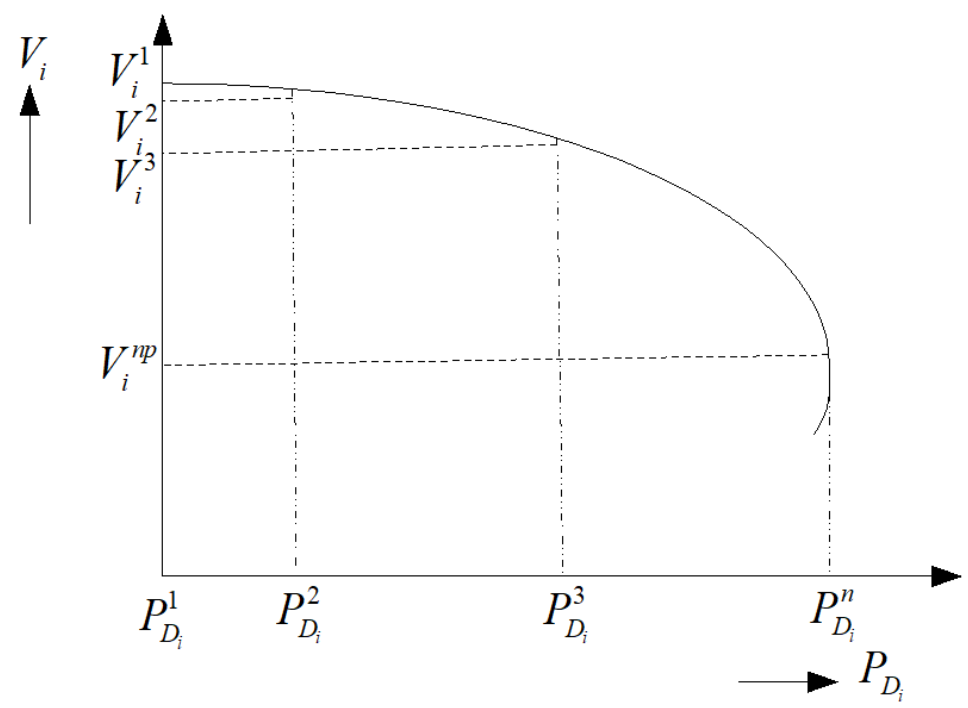

Figure 1. P-V curve of bus-i

Reactive power demand $\left(Q_{D_{i}}\right)$ versus voltage magnitude $\left(V_{i}\right)$ curve ( $Q$ - $V$ curve) of bus- $i$ shown in Figure 2 may be approximately obtained by solution of quadratic equation,

$$
Q_{D_{i}}=b_{1 i} V_{i}^{2}+b_{2 i} V_{i}+b_{3 i}
$$

where, $b_{1 i}, b_{2 i}$ and $b_{3 i}$ are constants. 


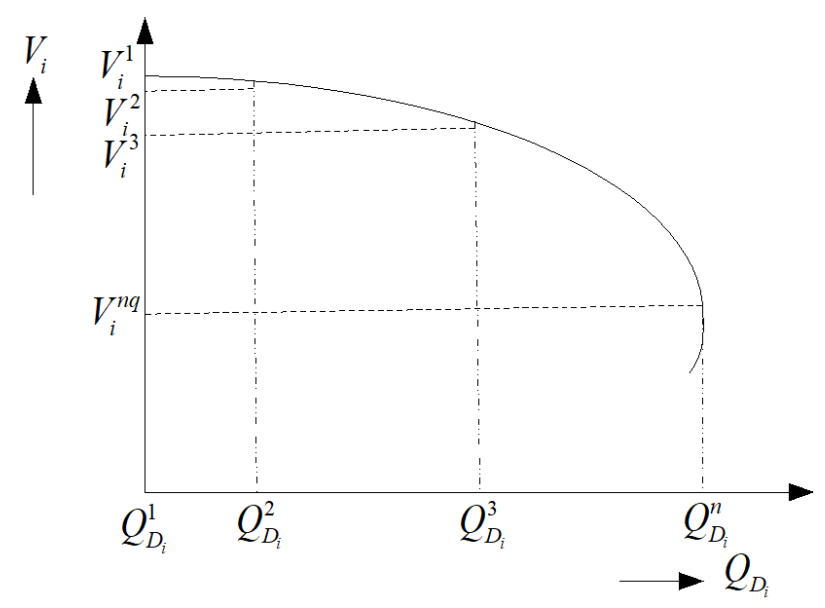

Figure 2. Q-V curve of bus-i

Differentiating $Q_{D_{i}}$ with respect to $V_{i}$,

$$
\frac{d Q_{D_{i}}}{d V_{i}}=2 b_{1 i} V_{i}+b_{2 i}
$$

At the nose point of $Q-V$ curve, $\frac{d Q_{D_{i}}}{d V_{i}}=0$, Therefore, from (6),

$$
V_{i}^{n q}=-\frac{b_{2 i}}{2 b_{1 i}}
$$

where, $V_{i}^{n q}=$ voltage magnitude of bus- $i$ at the nose point of $Q$ - $V$ curve shown in Figure 2. From (5) and (7),

$$
Q_{D_{i}}^{n}=-\frac{b_{2 i}^{2}}{4 b_{1 i}}+b_{3 i}
$$

where, $Q_{D_{i}}^{n}=$ Reactive power demand of bus- $i$ at the nose point of $Q-V$ curve shown in Figure 2. Constants $a_{1 i}, a_{2 i}$ and $a_{3 i}$ were obtained by solution of equations:

$$
\begin{aligned}
& P_{D_{i}}^{1}=a_{1 i}\left(V_{i}^{1}\right)^{2}+a_{2 i} V_{i}^{1}+a_{3 i} \\
& P_{D_{i}}^{2}=a_{1 i}\left(V_{i}^{2}\right)^{2}+a_{2 i} V_{i}^{2}+a_{3 i} \\
& P_{D_{i}}^{3}=a_{1 i}\left(V_{i}^{3}\right)^{2}+a_{2 i} V_{i}^{3}+a_{3 i}
\end{aligned}
$$

where, $V_{i}^{1}, V_{i}^{2}, V_{i}^{3}$ shown in Figure 1 and in Figure 2 correspond to voltage magnitude of bus- $i$ at operating points 1,2 and 3 , respectively, and $P_{D_{i}}^{1}, P_{D_{i}}^{2}$ and $P_{D_{i}}^{3}$ shown in Figure 1 correspond to real power demand of bus- $i$ at operating points 1,2 and 3, respectively.

Evaluated constants $a_{1 i}, a_{2 i}$ and $a_{3 i}$ were used to find real power loading margin of bus- $i$ using (4). Constants $b_{1 i}, b_{2 i}$ and $b_{3 i}$ are obtained by solution of equations:

$$
Q_{D_{i}}^{1}=b_{1 i}\left(V_{i}^{1}\right)^{2}+b_{2 i} V_{i}^{1}+b_{3 i}
$$




$$
\begin{aligned}
& Q_{D_{i}}^{2}=b_{1 i}\left(V_{i}^{2}\right)^{2}+b_{2 i} V_{i}^{2}+b_{3 i} \\
& Q_{D_{i}}^{3}=b_{1 i}\left(V_{i}^{3}\right)^{2}+b_{2 i} V_{i}^{3}+b_{3 i}
\end{aligned}
$$

where, $Q_{D_{i}}^{1}, Q_{D_{i}}^{2}$ and $Q_{D_{i}}^{3}$ shown in Figure 2 correspond to reactive power demand of bus- $i$ at operating points 1,2 and 3 , respectively.

Evaluated constants $b_{1 i}, b_{2 i}$ and $b_{3 i}$ were used to find reactive power loading margin of bus- $i$ using (8). Constants $a_{l i}, a_{2 i}, a_{3 i}, b_{l i}, b_{2 i}$ and $b_{3 i}$ for each of the load buses were evaluated using voltage magnitude, real power demand and reactive power demand obtained by PMU measurements/pseudo measurements performed at operating points 1, 2 and 3, respectively. Evaluated constants predict real power loading margin as well as reactive power loading margin of each bus using (4) and (8), respectively. Minimum out of maximum real power loadability of all the load buses present in the system is considered as real power loading margin of the system, and corresponding bus was considered as the most critical bus based on real power loading margin. Minimum out of maximum reactive power loadability of all the load buses present in the system was considered as reactive power loading margin of the system, and corresponding bus was considered as the most critical bus based on reactive power loading margin criterion. A flow chart for finding loading margin as well as most critical bus based on PMU measurements is shown in Figure 3. Since, loading margin of a real time system keeps on changing with change in operating conditions; it is proposed to update loading margin as well as most critical bus information based on new PMU measurements obtained, at regular intervals. Flowchart shown in Figure 3 assumes very high initial loading margin of 10,000 MW and 10,000 MVAR, respectively, keeping in mind such values to be higher than loading margin of any of the load buses present in the system, and keeps on reducing these till real power loading margin as well as reactive power loading margin of the most critical bus are obtained. Pseudo measurements were performed using following network observability rules:

a. If voltage and current phasor at one end of a branch are known, voltage phasor at the other end of the branch can be calculated using Ohm's law.

b. If voltage phasors at both the ends of a branch are known, branch current can be calculated

c. If there exists a zero-injection bus with all branch currents known except for one, the unknown branch current can be calculated using Kirchhoff's Current Law (KCL).

\section{CASE STUDIES}

Proposed approach of online monitoring of voltage stability margin was tested on IEEE 14-bus system, New England 39-bus system and 246-bus Northern Region Power Grid (NRPG) system of India with the help of Power System Analysis Toolbox (PSAT) software [24]. Simulation results obtained on three systems are presented below:

\subsection{IEEE 14-Bus System}

IEEE 14-bus system consists of two synchronous generators (at bus numbers 1 and 2), three synchronous condensers (at bus number 3,6 and 8) and 20 transmission lines including three transformers [25]. This system has a zero-injection bus at bus number 7. PMUs were placed at bus numbers 2, 4, 5, 6 and 9 based on results of binary integer linear programming [23] ensuring full network observability even in case of loss of few PMUs. Voltage magnitude, real power demand and reactive power demand of all the load buses were obtained at three operating points (viz. points 1,2 and 3, respectively) using combination of PMU measurements and pseudo measurements for the system intact case. Constants $a_{l i}$, $a_{2 i}$ and $a_{3 i}$ were calculated using (9), (10) and (11) for each of the load buses. Evaluated constants were used to find nose point real power demand $\left(P_{D_{i}}^{n}\right)$ of each load bus using (4). Minimum out of nose point real power demand of all the load buses was considered as real power loading margin $P_{D}^{M a x}$ of the intact system, and bus having minimum $P_{D_{i}}^{n}$ value was considered as the most critical bus requiring attention as far as system real power loadability is concerned. In order to update loading margin information under change in operating scenario, PMU measurements as well as pseudo measurements obtained at three operating points under all the single line outage cases were used for evaluation of updated $a_{1 i}, a_{2 i}, a_{3 i}$ for all the load buses, and new $P_{D}^{M a x}$ were calculated under these conditions using flow chart shown in Figure 3. 

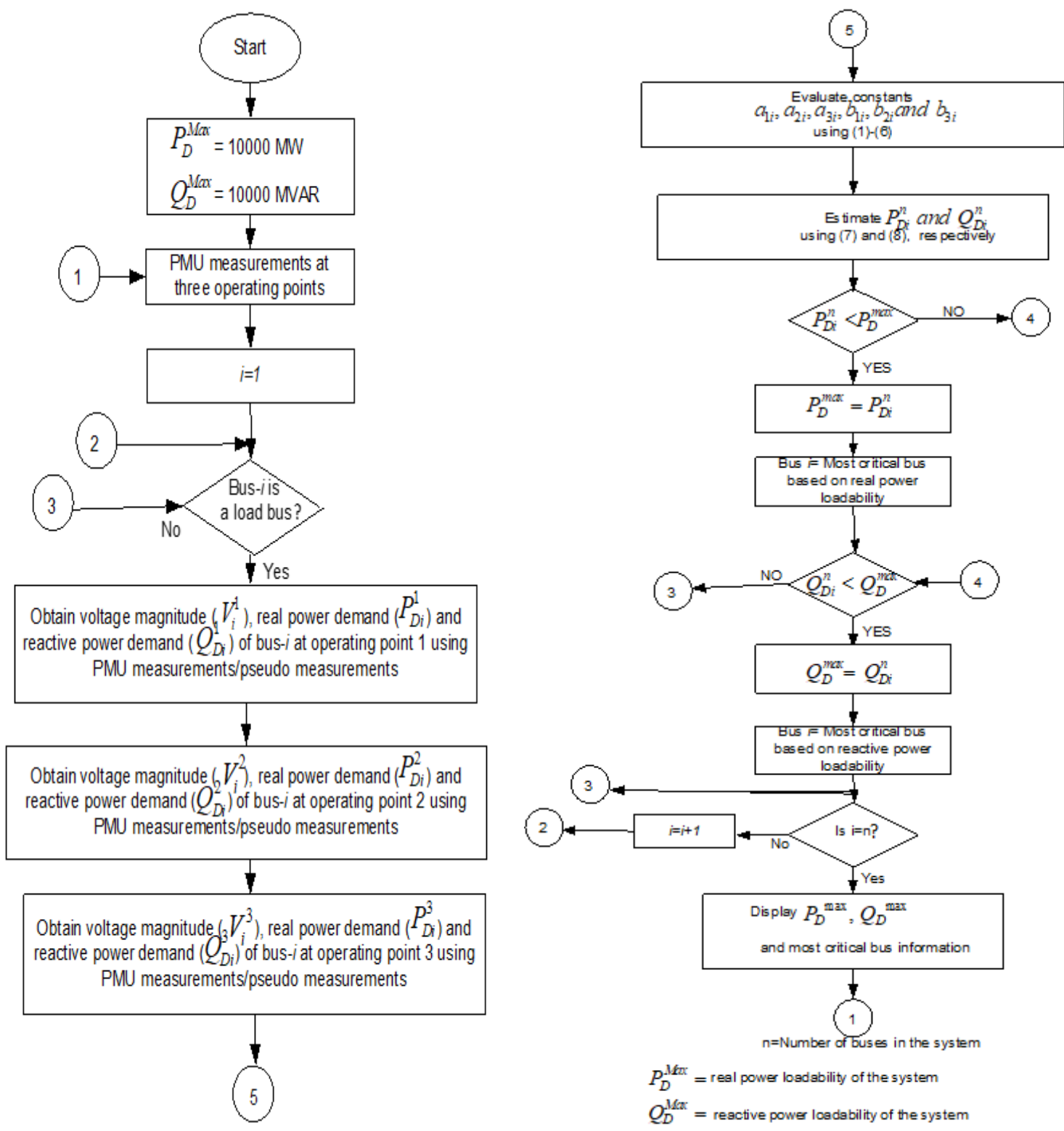

Figure 3. Flowchart for determining loading margin of system using proposed approach

Measured voltage magnitude and real power demand of the most critical bus at the three operating points, $P_{D}^{\text {Max }}$ calculated using proposed approach and real power loadability based most critical bus number have been shown in Table 1 for the system intact case and few critical contingency cases. In order to validate real power loading margin obtained by proposed approach, real power demand versus voltage magnitude curve $(P-V$ curve) of most critical bus was plotted using continuation power flow (CPF) method [26] for the system intact case and all the single line outage cases. For obtaining $P-V$ curve of the most critical bus using CPF method, its real power demand $P_{D_{j}}$ was varied as per following:

$$
P_{D j}=P_{D_{j}}^{1}\left(1+\lambda_{j p}\right)
$$

where, $\lambda_{j p}=$ fraction of real power demand increase at bus- $j$

Real power loading margin $\left(P_{D}^{M a x}\right)$ of the most critical bus obtained by CPF method (real power demand at the nose point of its $P$ - $V$ curve) have also been shown in Table 1 for the system intact case and few critical contingency cases. It is observed from Table 1 that real power loading margin obtained by proposed approach closely matches with real power loading margin found by continuation power flow method. 
Table 1. Real power loading margin under critical contingencies (IEEE 14-bus system)

\begin{tabular}{|c|c|c|c|c|c|c|c|c|c|}
\hline \multirow{2}{*}{ C.C. } & \multirow{2}{*}{$\begin{array}{l}\text { M.C.B } \\
\text { (Bus-j) }\end{array}$} & \multicolumn{2}{|c|}{ Point 1} & \multicolumn{2}{|c|}{ Point 2} & \multicolumn{2}{|c|}{ Point 3} & \multicolumn{2}{|c|}{$P_{D}^{M a \mathrm{x}}(\mathrm{MW})$} \\
\hline & & $V_{j}^{1}$ (p.u.) & $P_{D_{j}}^{1}(\mathrm{MW})$ & $V_{j}^{2}$ (p.u.) & $P_{D_{j}}^{2}(\mathrm{MW})$ & $V_{j}^{3}$ (p.u.) & $P_{D_{j}}^{3}(\mathrm{MW})$ & P.A. & $\mathrm{CPF}$ \\
\hline Intact & 5 & 1.03 & 7.60 & 0.96 & 36.16 & 0.90 & 37.09 & 39.44 & 40.20 \\
\hline $1-2$ & 5 & 1.03 & 7.60 & 0.95 & 17.71 & 0.91 & 16.26 & 17.78 & 16.49 \\
\hline $2-3$ & 4 & 1.03 & 47.8 & 0.95 & 177.82 & 0.90 & 187.85 & 189.7 & 188.3 \\
\hline $2-4$ & 5 & 1.03 & 7.60 & 0.96 & 30.40 & 0.90 & 30.86 & 32.76 & 32.91 \\
\hline $1-5$ & 5 & 1.02 & 7.60 & 0.95 & 37.32 & 0.92 & 34.50 & 37.39 & 34.50 \\
\hline $2-5$ & 5 & 1.02 & 7.60 & 0.95 & 33.14 & 0.90 & 33.21 & 35.64 & 35.26 \\
\hline
\end{tabular}

$P-V$ curve of bus 4 (most critical bus) obtained by proposed quadratic fitting of nose curves using PMU measurements/pseudo measurements at three operating points as well as continuation power flow based $P-V$ curve of same bus have been shown in Figure 4 for the outage of line 2-4. It is observed from Figure 4 that nose point real power loadability obtained by proposed approach closely matches with real power loading margin of the bus obtained by continuation power flow method.

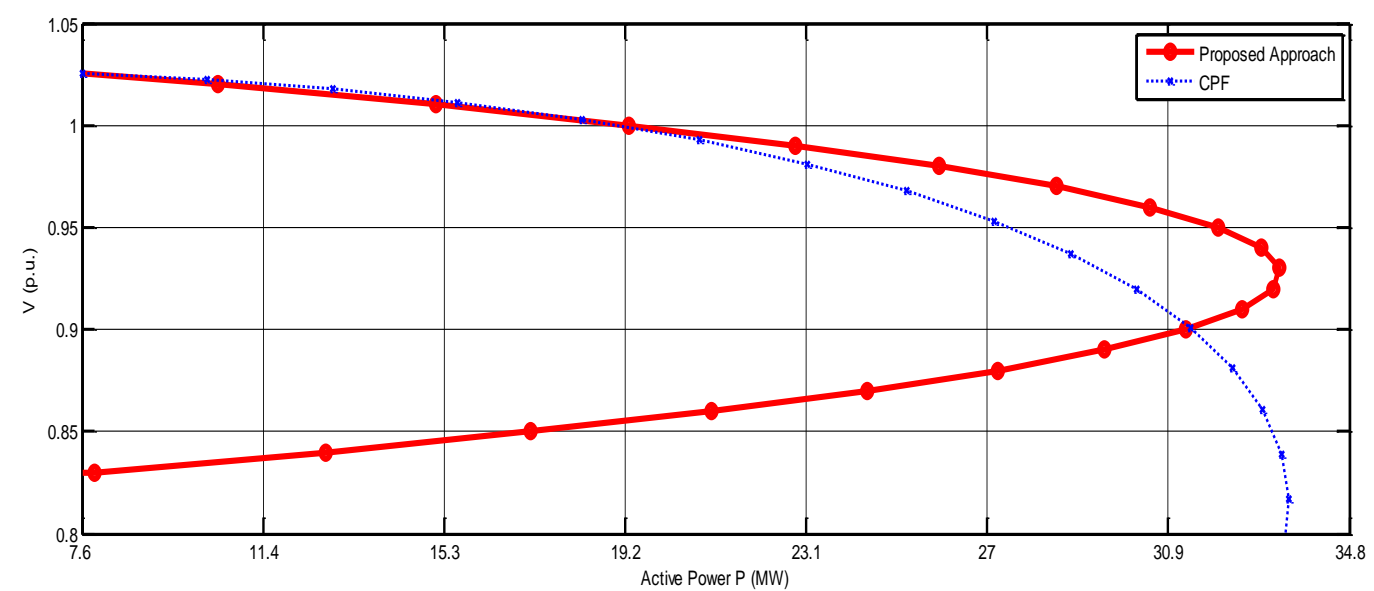

Figure 4. P-V curve of critical bus 5 obtained using proposed approach and by CPF method for line outage 2-4 (IEEE 14-bus system)

Constants $b_{1 i}, b_{2 i}$ and $b_{3 i}$ were calculated for each of the load buses using (12), (13) and (14) for the system intact case and all the single line outage cases using PMU measurements/pseudo measurements. Evaluated constants were utilized to determine nose point reactive power demand, $Q_{D_{i}}^{n}$ of each bus using (8). Minimum out of nose point reactive power demand $\left(Q_{D_{i}}^{n}\right)$ of all the load buses was considered as reactive power loading margin $Q_{D}^{\text {Max }}$ of the system, and bus having minimum $Q_{D_{i}}^{n}$ value was considered as most critical bus requiring attention as for as reactive power loading margin is concerned. In order to validate reactive power loading margin obtained by proposed approach, reactive power demand versus voltage magnitude curve ( $Q$ - $V$ curve) of the most critical bus was obtained by CPF method for the system intact case and all the single line outage cases. For obtaining $Q-V$ curve of the most critical bus using CPF method, its reactive power demand was varied using:

$$
Q_{D_{j}}=Q_{D_{j}}^{1}\left(1+\lambda_{j q}\right)
$$

where, $\lambda_{j q}=$ fraction of reactive power demand increase at bus- $j$

Measured voltage magnitude and reactive power demand of most critical bus at three operating points, reactive power loading margin $\left(Q_{D}^{M a x}\right)$ obtained by proposed approach as well as by CPF method have been shown in Table 2, for the system intact case and few critical contingency cases. Reactive power 
loadability based most critical bus number has also been shown in Table 2 for all these cases. It is observed from Table 2 that $Q_{D}^{M a x}$ obtained by proposed approach closely matches with $Q_{D}^{\text {Max }}$ obtained by CPF method.

Table 2. Reactive power loading margin under critical contingencies (IEEE 14-bus system)

\begin{tabular}{|c|c|c|c|c|c|c|c|c|c|}
\hline \multirow[t]{2}{*}{ C.C } & \multirow[t]{2}{*}{$\begin{array}{l}\text { M.C.B. } \\
\text { (Bus-j) }\end{array}$} & \multirow{2}{*}{$\begin{array}{l}\text { Point } 1 \\
V_{j}^{1} \text { (p.u.) }\end{array}$} & \multirow{2}{*}{$\begin{array}{c}\text { Point } 2 \\
Q_{D_{j}}^{1}(\mathrm{MVAR})\end{array}$} & \multirow{2}{*}{$\begin{array}{l}\text { Point } 3 \\
V_{j}^{2} \text { (p.u.) }\end{array}$} & \multicolumn{5}{|c|}{$Q_{D}^{\operatorname{Max}}$ (MVAR) } \\
\hline & & & & & $Q_{D_{j}}^{2}$ (MVAR) & $V_{j}^{3}$ (р.u.) & $Q_{D_{j}}^{3}$ (MVAR) & P.A. & $\mathrm{CPF}$ \\
\hline Intact & 5 & 1.03 & 0.32 & 0.96 & 3.81 & 0.90 & 7.81 & 0.85 & 0.86 \\
\hline $1-2$ & 5 & 1.03 & 0.32 & 0.95 & 1.86 & 0.91 & 3.42 & 0.56 & 0.54 \\
\hline $2-3$ & 4 & 1.03 & 0.78 & 0.95 & 7.25 & 0.90 & 15.33 & 3.10 & 3.07 \\
\hline $6-13$ & 13 & 1.01 & 1.16 & 0.95 & 3.20 & 0.91 & 6.50 & 5.57 & 6.04 \\
\hline $9-14$ & 14 & 1.02 & 1.00 & 0.96 & 3.93 & 0.92 & 7.26 & 4.68 & 5.22 \\
\hline $9-10$ & 10 & 1.02 & 1.16 & 0.96 & 3.49 & 0.91 & 6.99 & 5.64 & 6.10 \\
\hline
\end{tabular}

$Q$ - $V$ curve of bus 4 (most critical bus) obtained by proposed quadratic curve fitting of nose curves using PMU measurements/pseudo measurements obtained at three operating points as well as CPF based $Q-V$ curve of same bus have been shown in Figure 5 for the outage of line 2-3. It is observed from Figure 5 that nose point reactive power loadability of bus 4 obtained by proposed approach closely matches with CPF based nose point reactive power demand.

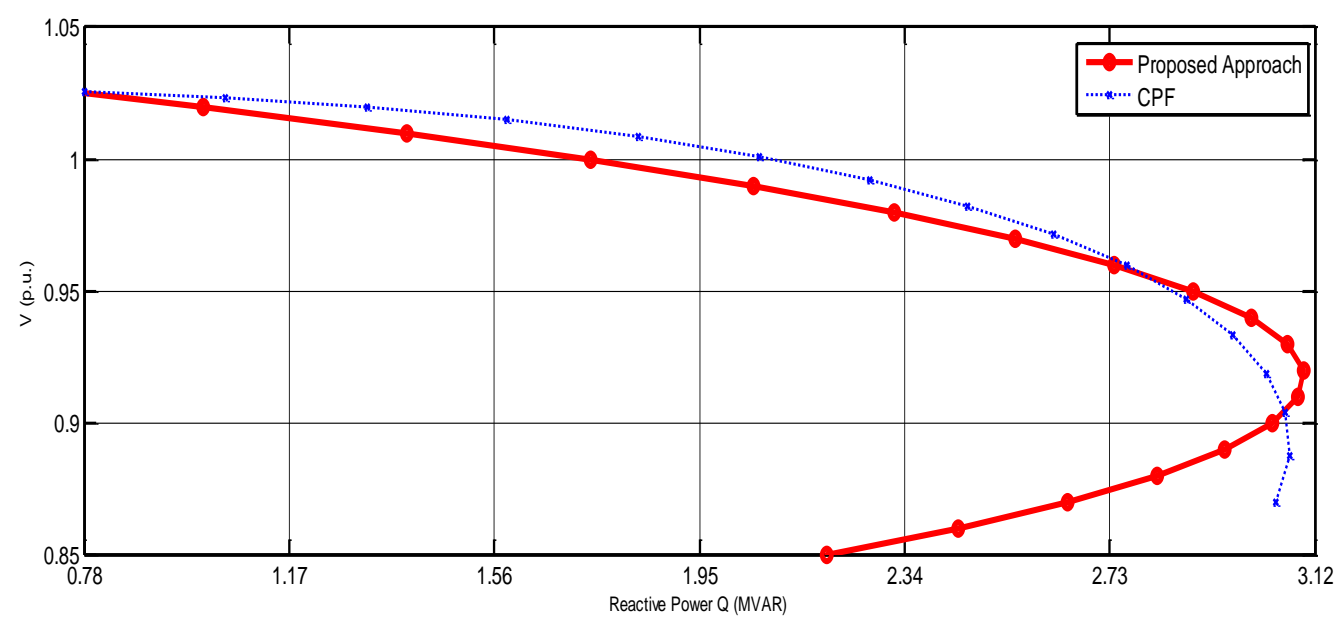

Figure 5. Q-V curve of critical bus 4 obtained using proposed approach and by CPF method for line outage 2-3 (IEEE 14-bus system)

\subsection{New England 39-Bus System}

The New England 39-Bus System has 10 generators and 46 transmission lines with 12 zero-injection buses at bus numbers 1, 2, 5, 6, 9, 10,11, 13, 14, 17, 19 and 22 [27]. Binary integer linear programming was used to optimally place PMUs in the system ensuring full network observability even in case of loss of few PMUs [23]. It results PMU placement at bus numbers 4, 8, 12, 16, 18, 20, 23, 25, 26, 27, 29, 30, 31, 32, 33, $34,35,36,37,38$ and 39.

Real power loading margin $P_{D}^{\operatorname{Max}}$ was calculated as per flow chart shown in Figure 3 for the system intact case and all the single line outage cases, as in case of IEEE 14-bus system. Measured voltage magnitude and real power demand of the most critical bus at the three operating points, $P_{D}^{\operatorname{Max}}$ calculated using proposed approach and real power loadability based most critical bus number have been shown in Table 3 for the system intact case and few critical contingency cases. In order to validate real power loadability obtained by proposed approach, real power demand versus voltage magnitude curve $(P-V$ curve) of most critical bus was plotted using continuation power flow (CPF) method [26] for the system intact case and all the single line outage cases. For obtaining $P-V$ curve of the most critical bus, its real power demand was varied as per (15). Real power loading margin $\left(P_{D}^{M a x}\right)$ of the most critical bus obtained by CPF 
method (real power demand at the nose point of its $P$ - $V$ curve) have also been shown in Table 3 for the system intact case and few critical contingency cases. It is observed from Table 3 that real power loading margin obtained by proposed approach closely matches with real power loading margin found by continuation power flow method.

Table 3. Real power loading margin under critical contingencies (New England 39-bus system)

\begin{tabular}{cccccccccc}
\hline C.C. & $\begin{array}{c}\text { M.C.B } \\
\text { (Bus-j) }\end{array}$ & Point 1 & Point 2 & Point 3 & \multicolumn{5}{c}{$P_{D}^{M a x}(\mathrm{MW})$} \\
& & $V_{j}^{1}$ (p.u.) & $P_{D_{j}}^{1}(\mathrm{MW})$ & $V_{j}^{2}$ (p.u.) & $P_{D_{j}}^{2}(\mathrm{MW})$ & $V_{j}^{3}$ (p.u.) & $P_{D_{j}}^{3}(\mathrm{MW})$ & P.A. & CPF \\
\hline Intact & 29 & 1.03 & 283.5 & 0.96 & 1227.56 & 0.90 & 1360.80 & 1363.64 & 1686.83 \\
$28-29$ & 29 & 1.02 & 283.5 & 0.95 & 768.29 & 0.89 & 853.34 & 856.17 & 989.42 \\
$29-38$ & 20 & 1.00 & 680 & 0.97 & 2380 & 0.96 & 2380 & 2420.8 & 2380 \\
$21-22$ & 23 & 1.05 & 247.5 & 0.95 & 868.73 & 0.91 & 905.85 & 908.33 & 930.60 \\
$22-35$ & 29 & 1.03 & 283.5 & 0.98 & 1097.15 & 0.96 & 1097.15 & 1108.49 & 1099.98 \\
$10-32$ & 29 & 1.03 & 283.5 & 0.98 & 1102.82 & 0.96 & 1102.82 & 1114.16 & 1102.82 \\
\hline
\end{tabular}

$P-V$ curve of bus 20 (most critical bus) obtained by proposed quadratic fitting of nose curves using PMU measurements/pseudo measurements obtained at three operating points as well as continuation power flow based $P-V$ curve of same bus have been shown in Figure 6 for the outage of line 29-38. It is observed from Figure 6 that nose point real power loadability obtained by proposed approach closely matches with real power loading margin of the bus obtained by continuation power flow method.

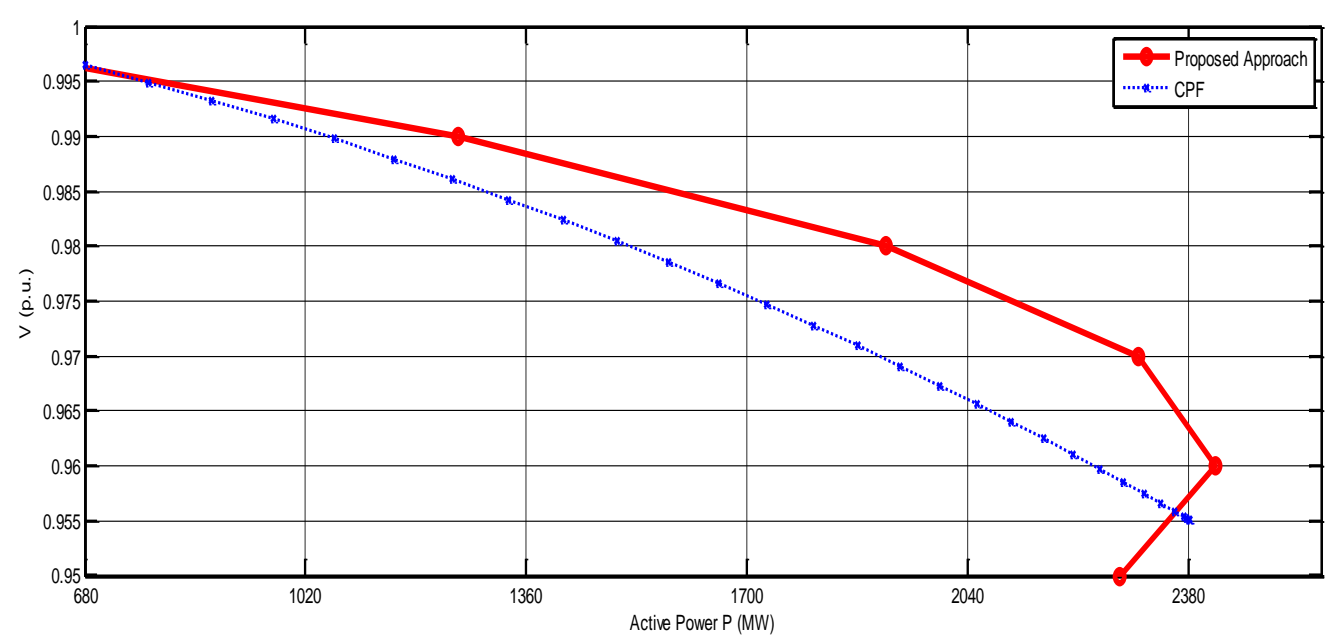

Figure 6. P-V curve of critical bus 20 obtained using proposed approach and by CPF method for line outage 29-38 (New England 39-bus system)

Reactive power loading margin $\left(Q_{D}^{\operatorname{Max}}\right)$ was calculated as per flow chart shown in Figure 3 for the system intact case and all the single line outage cases, as in case of IEEE 14-bus system. In order to validate reactive power loading margin obtained by proposed approach, reactive power demand versus voltage magnitude curve $(Q-V$ curve) of the most critical bus was also obtained by CPF method for the system intact case and all the single line outage cases. For obtaining $Q$ - $V$ curve of the most critical bus, its reactive power demand was varied as per (16). Measured voltage magnitude and reactive power demand of most critical bus at three operating points, reactive power loading margin $\left(Q_{D}^{M a x}\right)$ obtained by proposed approach as well as by CPF method have been shown in Table 4, for the system intact case and few critical contingency cases. Reactive power loadability based most critical bus number has also been shown in Table 4 for all these cases. It is observed from Table 4 that $Q_{D}^{\text {Max }}$ obtained by proposed approach closely matches with $Q_{D}^{M a x}$ obtained by CPF method. 
Table 4. Reactive power loading margin under critical contingencies (New England 39-bus system)

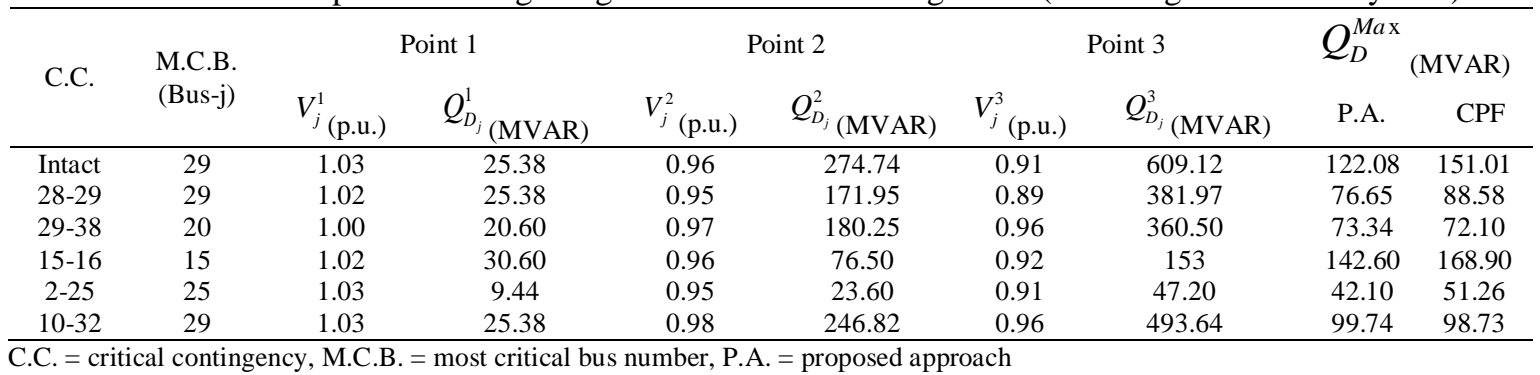

$Q-V$ curve of bus 29 (most critical bus) obtained by proposed quadratic curve fitting of nose curves using PMU measurements/pseudo measurements at three operating points as well as CPF based $Q$ - $V$ curve of same bus have been shown in Figure 7 for the outage of line 10-32. It is observed from Figure 7 that nose point reactive power loadability of bus 29 obtained by proposed approach closely matches with CPF based nose point reactive power demand.

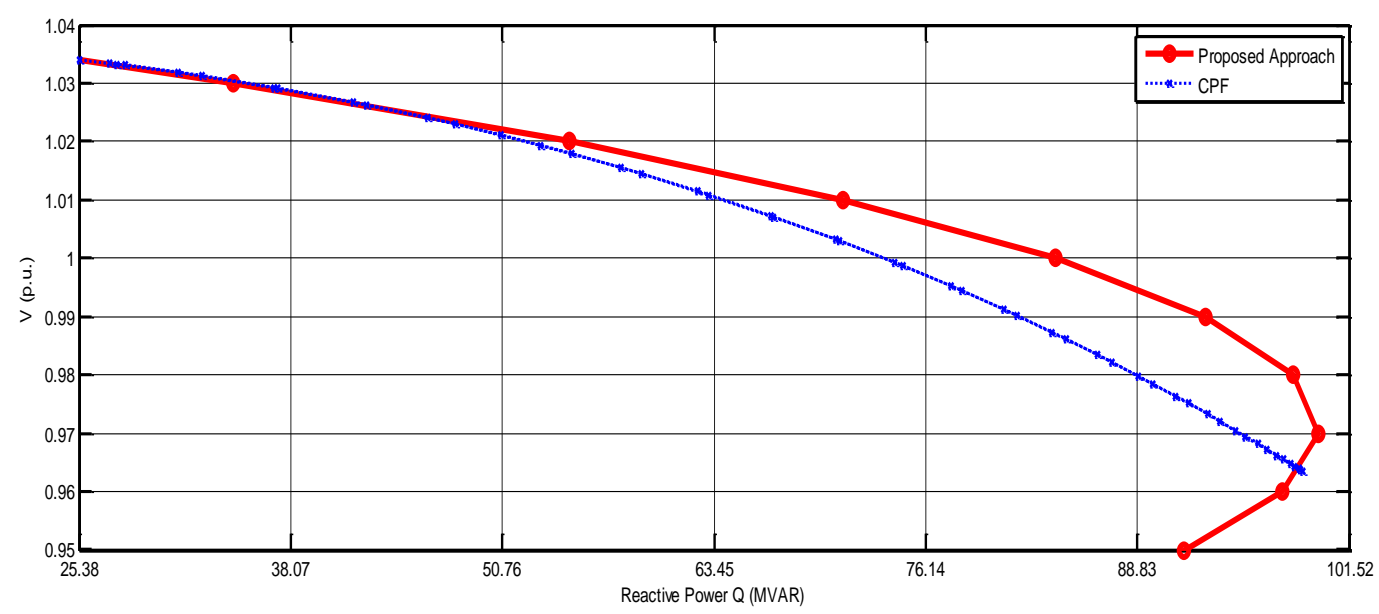

Figure 7. Q-V curve of critical bus 29 obtained using proposed approach and by CPF method for line outage 10-32 (New England 39-bus system)

\subsection{NRPG 246-Bus System}

The 246-bus Northern Regional Power Grid (NRPG) system covers power network of seven states (Jammu and Kashmir, Himachal Pradesh, Punjab, Haryana, Rajasthan, Uttarakhand and Uttar Pradesh) and two union territories (Chandigarh and New Delhi) of India [28]. It consists of 42 generators, 36 transformers and 376 transmission lines. It has 15 zero-injection buses at bus numbers 63, 75, 81, 102, 103, 104, 107, 122 , 155, 180, 210, 226, 237, 241, and 244. Binary Integer linear Programming was run to optimally place 97 PMUs in the 246-bus NRPG system to ensure complete observability even in case of loss of few PMUs [23]. $P_{D}^{M a x}$ was calculated for the system as per flow chart shown in Figure 3 for the system intact case and all the single line outage cases, as in case of IEEE 14-bus system and New England 39-bus system. Measured voltage magnitude and real power demand of the most critical bus at the three operating points, real power loadability based most critical bus number and $P_{D}^{M a x}$ calculated using proposed approach have been shown in Table 5 for the system intact case and few critical contingency cases. In order to validate real power loading margin obtained by proposed approach, real power demand versus voltage magnitude curve $(P-V$ curve) of most critical bus was plotted using continuation power flow (CPF) method [26] for the system intact case and all the single line outage cases. CPF based $P-V$ curve was obtained by varying real power demand at the bus as per (15). Real power loading margin $\left(P_{D}^{M a x}\right)$ of the most critical bus obtained by CPF method (real power demand at the nose point of its $P$ - $V$ curve) have also been shown in Table 5 for the system intact case and few critical contingency cases. It is observed from Table 5 that real power loading margin obtained by proposed approach closely matches with real power loading margin found by continuation power flow method. 
$P-V$ curve of bus 174 (most critical bus) obtained by proposed quadratic fitting of nose curves using PMU measurements/pseudo measurements at three operating points as well as continuation power flow based $P-V$ curve of same bus have been shown in Figure 8 for the outage of line 194-198. It is observed from Figure 8 that nose point real power loadability obtained by proposed approach closely matches with real power loading margin of the bus obtained by continuation power flow method.

$Q_{D}^{\operatorname{Max}}$ was calculated for the system intact case and all the single line outage cases, as in case of IEEE 14-bus system and New England 39-bus system. In order to validate reactive power loading margin obtained by proposed approach, reactive power demand versus voltage magnitude curve ( $Q$ - $V$ curve) of the most critical bus was also obtained by CPF method for the system intact case and all the single line outage cases. CPF based $Q-V$ curve was obtained by varying reactive power demand at the bus as per (16). Measured voltage magnitude and reactive power demand of most critical bus at three operating points, reactive power loading margin $\left(Q_{D}^{M a x}\right)$ obtained by proposed approach as well as by CPF method have been shown in Table 6, for the system intact case and few critical contingency cases. Reactive power loadability based most critical bus number has also been shown in Table 6 for all these cases. It is observed from Table 6 that $Q_{D}^{M a x}$ obtained by proposed approach closely matches with $Q_{D}^{M a x}$ obtained by CPF method.

$Q-V$ curve of bus 158 (most critical bus) obtained by proposed quadratic curve fitting of nose curves using PMU measurements/pseudo measurements at three operating points as well as CPF based $Q-V$ curve of same bus have been shown in Figure9 for the outage of line 156-158. It is observed from Figure 9 that nose point reactive power loadability of bus 158 obtained by proposed approach closely matches with CPF based nose point reactive power demand.

Table 5. Real power loading margin under critical contingencies (NRPG 246-bus system)

\begin{tabular}{|c|c|c|c|c|c|c|c|c|c|}
\hline \multirow[t]{2}{*}{ C.C. } & \multirow{2}{*}{$\begin{array}{l}\text { M.C.B. } \\
\text { (Bus-j) }\end{array}$} & \multicolumn{2}{|c|}{ Point 1} & \multicolumn{2}{|c|}{ Point 2} & \multicolumn{2}{|c|}{ Point 3} & \multicolumn{2}{|c|}{$P^{M a \mathrm{x}}$} \\
\hline & & $V_{j}^{1}$ (p.u.) & $P_{D_{j}}^{1}(\mathrm{MW})$ & $V_{j}^{2}$ (p.u.) & $P_{D_{j}}^{2}(\mathrm{MW})$ & $V_{j}^{3}$ (p.u.) & $P_{D_{j}}^{3}(\mathrm{MW})$ & P.A. & $\mathrm{CPF}$ \\
\hline Intact & 174 & 1.01 & 169.8 & 0.95 & 419.41 & 0.90 & 485.63 & 487.33 & 641.84 \\
\hline $173-174$ & 174 & 1.01 & 169.8 & 0.96 & 249.61 & 0.90 & 264.89 & 269.98 & 344.69 \\
\hline $40-41$ & 174 & 1.01 & 169.8 & 0.96 & 382.05 & 0.94 & 384.35 & 388.84 & 383.75 \\
\hline $166-173$ & 174 & 1.01 & 169.8 & 0.95 & 339.60 & 0.90 & 383.70 & 385.45 & 434.69 \\
\hline $156-158$ & 158 & 1.01 & 174.7 & 0.97 & 468.20 & 0.96 & 459.46 & 473.44 & 476.93 \\
\hline $194-198$ & 174 & 1.01 & 174.7 & 0.95 & 468.20 & 0.90 & 459.46 & 506.63 & 518.86 \\
\hline
\end{tabular}

C.C $=$ critical contingency, M.C.B.=most critical bus number, P.A.=proposed approach

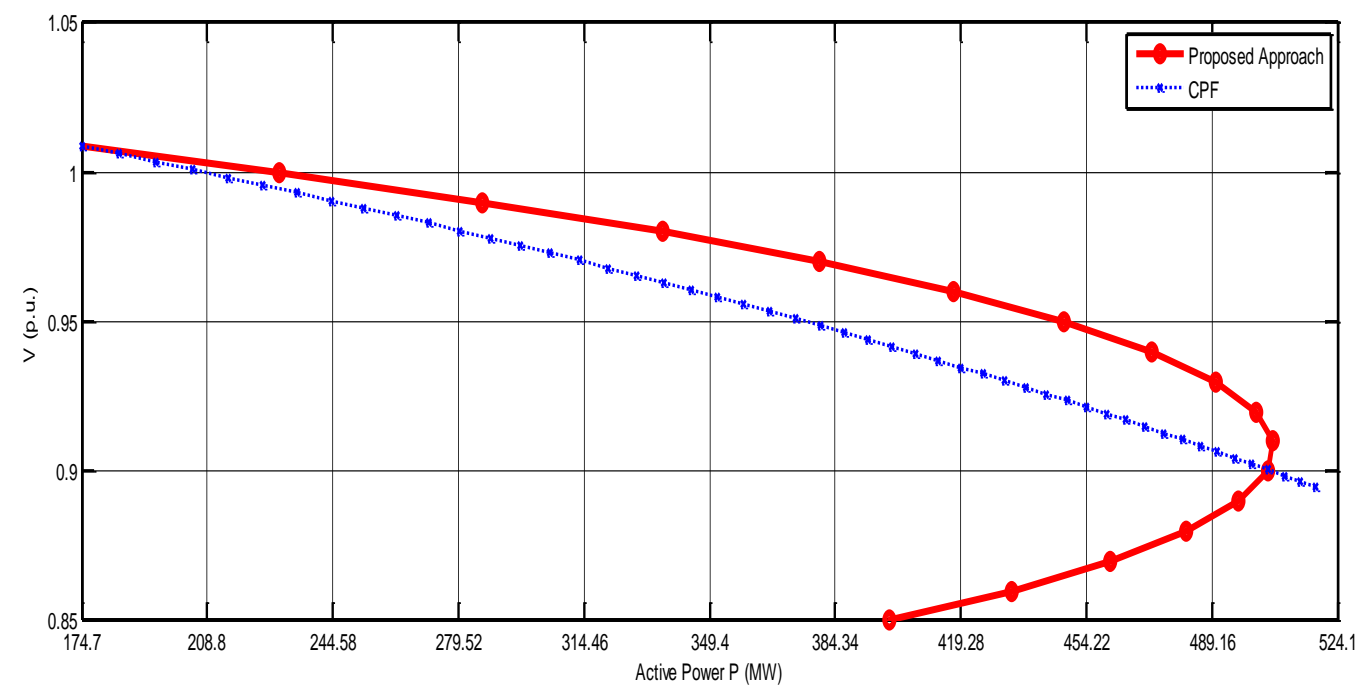

Figure 8. P-V curve of critical bus 174 obtained using proposed approach and by CPF method for line outage 194-198 (NRPG 246-bus system) 


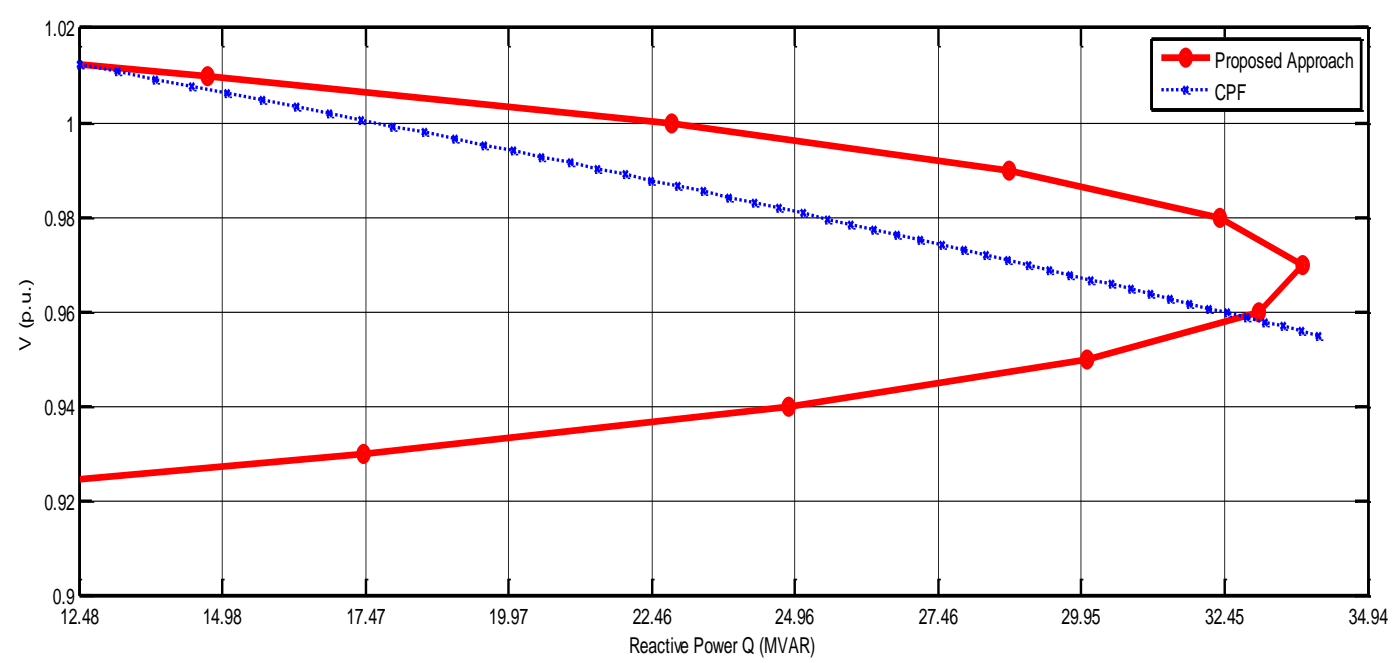

Figure 9. Q-V curve of critical bus 158 obtained using proposed approach and by CPF method for line outage 156-158 (NRPG 246-bus system)

Table 6. Reactive power loading margin under critical contingencies (NRPG 246-bus system)

\begin{tabular}{lccccccccc}
\hline \multicolumn{1}{c}{ C.C. } & $\begin{array}{c}\text { M.C.B. } \\
\text { (Bus-j) }\end{array}$ & \multicolumn{2}{c}{ Point 1 } & \multicolumn{2}{c}{ Point 2 } & \multicolumn{2}{c}{ Point 3 } & \multicolumn{2}{c}{$Q_{D}^{\text {Max }}$ (MVAR) } \\
& & $V_{j}^{1}$ (p.u.) & $Q_{D_{j}}^{1}$ (MVAR) & $V_{j}^{2}$ (p.u.) & $Q_{D_{j}}^{2}$ (MVAR) & $V_{j}^{3}$ (p.u.) & $Q_{D_{j}}^{3}$ (MVAR) & P.A. & CPF \\
\hline Intact & 174 & 1.01 & 13.52 & 0.95 & 83.49 & 0.90 & 193.34 & 38.80 & 51.11 \\
$173-174$ & 174 & 1.01 & 13.52 & 0.96 & 49.69 & 0.90 & 105.46 & 21.50 & 27.45 \\
$40-41$ & 174 & 1.01 & 13.52 & 0.96 & 76.05 & 0.94 & 151.42 & 30.96 & 30.56 \\
$166-173$ & 174 & 1.01 & 13.52 & 0.95 & 67.6 & 0.90 & 152.8 & 30.69 & 34.61 \\
$156-158$ & 158 & 1.01 & 12.48 & 0.97 & 50.86 & 0.96 & 164.11 & 33.82 & 34.07 \\
$63-70$ & 156 & 1.01 & 17.74 & 1.01 & 44.35 & 1.01 & 88.7 & 19.51 & 19.33 \\
\hline
\end{tabular}

C.C. $=$ critical contingency, M.C.B. $=$ most critical bus number, P.A. $=$ proposed approach

\section{CONCLUSION}

Online monitoring of voltage stability margin using PMU measurements has been proposed in this work. Proposed approach estimates voltage stability margin based on measurements obtained at three operating points. Due to highly dynamic nature of power systems, voltage stability margin keeps on changing. Therefore, proposed approach suggests computation of updated voltage stability margin at regular intervals based on new PMU measurements obtained. Change in operating scenario has been simulated in PSAT software considering different single line outage cases. Accuracy of proposed approach has been validated by comparing voltage stability margin obtained by proposed approach with margin estimated using continuation power flow method under same set of operating conditions. Case studies performed on three test systems show that real power loading margin as well as reactive power loading margin of the system obtained by proposed approach closely matches with loading margin obtained by continuation power flow method.

\section{REFERENCES}

[1] C. W. Taylor, Power system voltage stability, New York: Mc Graw Hill, 1994.

[2] P. Kundur, Power system stability and control, New York: Mc Graw Hill, 1994.

[3] V. Ajjarapu and B. Lee, "Bibliography on Voltage Stability," IEEE Trans. on Power Systems, pp. 115-125, vol. 13, no. 1 , Feb 1998.

[4] Antonio C. Zambroni de Souza et al, "On-Line Voltage Stability Monitoring," IEEE Trans. on Power Systems, pp. 1300-1305, vol. 15, no. 4, Nov 2000.

[5] Bruno Leonardi and Venkataramana Ajjarapu, "Development of Multi Linear Regression Models for Online Voltage Stability Margin Estimation,” IEEE Trans. on Power Systems, pp. 374-383, vol. 26, no. 1, Feb 2011.

[6] Yiwei Qiu, Hao Wu, Yongzhi Zhou and Yonghua Song, "Global Parametric Polynomial Approximation of Static Voltage Stability Region Boundaries," IEEE Trans. on Power Systems, pp. 2362-2371, vol. 32, no. 3, May 2017.

[7] M. Glavic and T. Van Cutsem, "Wide-Area Detection of Voltage Instability from Synchronized Phasor Measurements, Part I: Principle,” IEEE Trans. on Power Systems, pp. 1408-1416, vol. 24, no. 2, May 2009. 
[8] F. Aminifar et al, "Synchrophasor Measurement Technology in Power Systems: Panorama and State-of-the-art" IEEE Access, pp. 1607-1628, vol. 2, 2014.

[9] K. Vu et. al., "Use of Local Measurements to Estimate Voltage Stability Margin," IEEE Trans. on Power Systems, vol. 14, no. 3, pp. 1029-1035, Aug 1999.

[10] I. Simen, G. Verbic and P. Gubina, "Local Voltage Stability Index using Tellegen's Theorem," IEEE Trans. on Power Systems, vol. 21, no. 3, pp. 1267-1275, Aug 2006.

[11] H. Y. Su and C. W. Liu, "Estimating of Voltage Stability Margin using PMU Measurements," IEEE Trans. on Power Systems, vol. 31, no. 4, pp. 3221-3229, Jul 2016.

[12] Costas D. Vournas, Charalambos Lambrou and Panagiotis Mandoulidis, "Voltage Stability Monitoring From a Transmission Bus PMU," IEEE Trans. on Power Systems, vol. 32, no. 4, pp. 3266-3274, Jul 2017.

[13] P. Zhang, L. Min and J. Chen, "Measurement-based Voltage Stability Monitoring and Control," U.S. Patent 8.126 667, 2012.

[14] K. Sun, P. Zhang and L. Min, "Measurement-based Voltage Stability Monitoring and Control for Load Centers," EPRI Report No. 1017798, 2009.

[15] Fengkai Hu et al, "Measurement Based Real-Time Voltage Stability Monitoring for Load Areas," IEEE Trans. on Power Systems, vol. 31, no. 4, pp. 2787-2798, Jul 2016.

[16] John William Simpson-Porco and Francesco Bullo, "Distributed Monitoring of Voltage Collapse Sensitivity Indices," IEEE Trans. on Smart Grid, vol. 7, no. 4, pp. 1979-1988, Jul 2016.

[17] Haoen Li, Anjan Bose and V. Venkatasubramanian, "Wide-Area Monitoring and Optimization," IEEE Trans. on Smart Grid, vol. 7, no. 2, pp. 785-793, Mar 2016.

[18] Mariana Kamel, Abdelrahman A. Karrar and Ahmed H. Eltom, "Development and Application of a New Voltage stability Index for On-Line Monitoring and Shedding," IEEE Trans. on Power Systems, vol. 33, no. 2, pp. 1231-1241, Mar 2018.

[19] Debbie Q. Zhou, Udaya D. Annakkage and Athula D. Rajapakse, "Online Monitoring of Voltage Stability Margin using an Artificial Neural Network,” IEEE PES General Meeting, USA, 25-29 Jul 2010.

[20] Heng-Yi Su and Tzu-Yi Liu, "Enhanced-Online-Random-Forcast Model for Static Voltage Stability Assessment Using Wide Area Measurements," IEEE Trans. on Power Systems, vol. 33, no. 6, pp. 6696-6704, Nov 2018.

[21] E. Gonzaled et. al., "PMU based Voltage Stability Indices with Intermediate Load Corrections," IEEE Latin America Trans. vol. 16, no. 4, pp. 1172-1179, Apr 2018.

[22] Samuel C. Chevalier and Paul D. H. Hines, "Mitigating the Risk of Voltage Collapse Using Statistical Measures from PMU Data," IEEE Trans. on Power Systems, vol. 34, no. 1, pp. 120-128, Jan 2019.

[23] Pankaj Sahu and M. K. Verma, "Optimal Placement of PMUs in Power System Network for Voltage Stability Estimation under Contingencies," 6th IEEE International Conference on Computer Applications in Electrical Engineering-Recent Advances (CERA), Roorkee, India, pp. 365-370, 5-7 Oct 2017.

[24] PSAT Software Manual, [Online]. Available: http://faradayl.ucd.ie/psat.html.

[25] IEEE 14-Bus System, [Online]. Available: http://www.ee.washington.edu/research/pstca/pf14/pg_tca14bus.htm].

[26] V. Ajjarapu and C. Christy, "The Continuation Power Flow: A Tool for Steady State Voltage Stability Analysis," IEEE Trans. on Power Systems, vol. 7, no. 1, pp. 416-423, Feb 1992.

[27] New England 39-Bus System [Online]. Available: http://icseg.iti.illinois.edu/ieee-39-bus-system/]

[28] North Region Power Grid (NRPG) 246-Bus System; 2013 [Online]. Available: http://www.iitk.ac.in/eeold/facilities/Research_labs/Power_System/NRPG-DATA.pdf].

\section{BIOGRAPHIES OF AUTHORS}

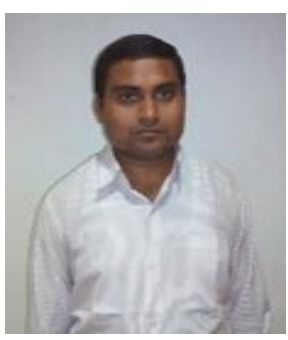

Pankaj Sahu received his B.Tech degree in electrical engineering from Harcourt Butler Technological Institute, Kanpur, India in 2007 and M.Tech degree in electrical engineering from Motilal Nehru National Institute of Technology, Allahabad, India in 2010. He is currently persuing for PhD degree at Indian Institute of Technology (BHU), Varanasi, India. His research interests include control system, instrumentation and voltage stability studies.

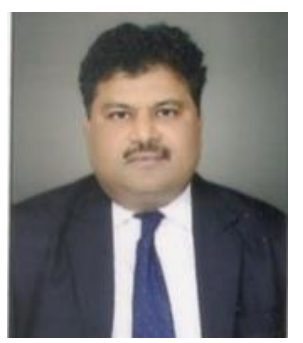

M. K. Verma received his B.Sc (Eng.) degree in electrical engineering from Regional Engineering College, Rourkela (presently National Institute of Technology, Rourkela), India in 1989, M.Sc (Eng.) degree in electrical engineering from Bihar Institute of Technology, Sindri, India in 1994 and $\mathrm{PhD}$ degree in electrical engineering from Indian Institute of Technology (IIT), Kanpur, India in 2005. He is currently working as Professor in electrical engineering department at Indian Institute of Technology (BHU), Varanasi, India. His current research interests include voltage stability studies, application of FACTS controllers, power quality, wide area monitoring system and smart grid 